\title{
Antioxidant Effects of Allium cepa and Cinnamon on Sex Hormones and Serum Antioxidant Capacity in Female Rats Exposed to Power Frequency Electric and Magnetic Fields
}

\author{
Elham Mansouri ${ }^{1}$, Ahmad Keshtkar ${ }^{1}$, Amir Afshin Khaki², Arash Khaki ${ }^{3}$
}

\begin{abstract}
Objectives: In this study we investigated whether $50 \mathrm{~Hz}$ power frequency electric field of $1.01 \times 10^{3} \mathrm{~V} / \mathrm{m}$ and the magnetic field flux density of $3 \mathrm{mT}$ have effect on serum sex hormones and antioxidant capacity and if Allium cepa and cinnamon can moderate potential adverse effects of power frequency electromagnetic exposure.

Materials and Methods: Forty female Wistar rats, weighting $220 \pm 10 \mathrm{~g}$ were used for this study. Rats were allocated randomly to four groups. The first group (the control group) received $3 \mathrm{cc}$ normal saline (0.9\%) daily and was treated for 6 weeks. The second group was exposed to power frequency of $3 \mathrm{mT}$ for $4 \mathrm{~h} /$ day, 7 days/week for 6 weeks. The third group received Allium cepa (3 cc onion juice) and cinnamon ( $75 \mathrm{mg} / \mathrm{kg}$ body weight) daily for 6 weeks by Gavage method. The fourth group was exposed to power frequency EMF of $3 \mathrm{mT}$ for $4 \mathrm{~h} /$ day, 7 days/week and received Allium cepa (3 cc onion juice) and cinnamon (75 mg/kg body weight) daily for 6 weeks by Gavage method.

Results: Levels of luteinizing hormone ( $\mathrm{LH})$, follicle-stimulating hormone (FSH) and estrogen, significantly decreased in second group that exposed to power frequency EMF of $3 \mathrm{mT}$ and $10^{3} \mathrm{~V} / \mathrm{m}(P<0.05)$ and modified in exposed rats which received Allium cepa and cinnamon $(P<0.05)$. Also, TAC levels were significantly decreased in exposed rats and were moderate in exposed rats received Allium cepa and cinnamon $(P<0.05)$.

Conclusion: Power frequency electromagnetic field could adversely affect sex hormones and total antioxidant capacity (TAC) levels in exposed rats and Allium cepa and cinnamon could prescribed as an effective pharmacological supplement to moderate exposure degenerative effects.

Keywords: Extremely low frequency, Electromagnetic fields, Follicle stimulating hormone (FSH), Luteinizing hormone (LH), Estrogens
\end{abstract}

\section{Introduction}

In addition to the background radiation of natural electromagnetic fields of the earth, human being is at the risk of manmade sources of electromagnetic field. Recently, with rapid development of new technologies, public concerns on potential effects of power frequency electromagnetic field on reproduction and fertility have been increased. Low electromagnetic fields of $1-300 \mathrm{~Hz}$ are commonly use and occur in power transmission lines, rail way power supply, transformers and in household power supply and devices $(1,2)$.

According to International Commission on Non-Ionizing Radiation Protection (ICNIRP) guideline, direct interact of external alternating low frequency magnetic field (power-frequency) could induce electric fields and circulating electric current flow within the human body. Also, the direct interaction of external alternative (power-frequency) electric field with the body could induce time-varying surface charge on the body results in induced currents in body. Each body tissue has finite conductivity. Magnitude of induced electric fields, currents and charges within body and depth of penetration depends on magnitude of the magnetic flux density and frequency of applied extremely low electromagnetic field $(3,4)$. The energy absorption and temperature rise induced by ELF-EMF in body are Inconsiderable. High internal current density results adverse biological effects on body. The induced current density by ELF electric field of $60 \mathrm{~Hz} 10^{3} \mathrm{~V} / \mathrm{m}$ in waist is $0.427 \times 10^{-3}(5)$.

In the range of extremely low frequency, electric and magnetic fields are measured separately because, in this range pure electric field $\mathrm{E}$ and almost pure magnetic $\mathrm{H}$ field could appear in some regions and $\mathrm{E}$ and $\mathrm{H}$ act independent and we could not reach to peak of both electric and magnetic fields in same location (6).

The International Agency for Research on Cancer (IARC) has classified ELF EMF as a $2 \mathrm{~B}$ possible carcinogen in humans and less than sufficient in animals in June 2001

Received 3 February 2016, Accepted 1 July 2016, Available online 1 July 2016

${ }^{1}$ Department of Medical Physics, School of Medicine, Tabriz University of Medical Sciences, Tabriz, Iran. ${ }^{2}$ Department of Anatomical Sciences, School of Medicine, Tabriz University of Medical Sciences, Tabriz, Iran. ${ }^{3}$ Women's Reproductive Health Research Center, Tabriz University of Medical Sciences, Tabriz, Iran.

*Corresponding author: Arash Khaki, Women’s Reproductive Health Research Center, Tabriz University of Medical Sciences, Tabriz, Iran. Tel: +98 9143138399; Email: arashkhaki@yahoo.com 
(7). Many studies have shown that exposure to extremely low electromagnetic fields can have adverse effects on reproductive system and tracts, and thus, a relationship between exposure to electromagnetic fields and infertility is conjectured (8).

Evidence showed that balance between reactive oxygen species (ROS) and biological antioxidants plays an important role in women's fertility and reproductive health (9). Electromagnetic fields have adverse effects on biological systems. Oxidative stress occurs as a result of increase in ROS concentrations in cells leading to various pathological damages $(10,11)$.

Cellular metabolisms and other exogenous environmental sources such as electromagnetic fields could cause ROS production in cells. High concentrations of ROS have been implicated to many infertility problems (12).

Dietary and pharmacological supplements were assumed to be helpful to reduce degenerative effects of ROS and free radicals on biological system. Antioxidants are chemical or biological agents that reduce oxidative damages. Antioxidant capacity white Allium cepa is rich in phenolic compounds in different layers and has high antioxidant activity $(13,14)$. Anti-oxidative and anti-mutagenic properties of cinnamon as a rich source of polyphenolic compounds have been indicated. Cinnamon could modify oxidative stress induced by different factors and has long been prescribed to cure fertility problems and other diseases (15).

The aim of this study was to evaluate adverse effects of 50 $\mathrm{Hz}, 3 \mathrm{mT}$ power frequency EMF on serum LH, FSH and estrogen and total antioxidant capacity (TAC) levels and if Allium cepa and cinnamon can reduce adverse effects of electromagnetic exposure.

\section{Materials and Methods}

Animals

Forty female Wistar rats, weighting $220 \pm 10 \mathrm{~g}$ were used for this study. Rats were 8-9 weeks old and were quarantined for 1 week maintained under standard conditions with respect to humidity (35\% to $60 \%$ ), illumination (12 hours light and 12 hours by dark fluorescent light controlling) and temperature $\left(20 \pm 2^{\circ} \mathrm{C}\right)$.

Rats were allocated randomly to four groups. A control group $(\mathrm{n}=10)$ and three treatment groups (total $=30)$ and $\mathrm{n}=10$ in each.

The first group (the control group) received $3 \mathrm{cc}$ normal saline $(0.9 \%)$ daily and was treated for 6 weeks.

The second group was exposed to ELF-EMF of $3 \mathrm{mT}$ for 4 h/day, 7 days/week for 6 weeks.

The third group received Allium cepa (3 cc onion juice) and cinnamon (75 mg/kg body weight) daily for 6 weeks by Gavage method.

The fourth group was exposed to ELF-EMF of $3 \mathrm{mT}$ for 4 h/day, 7 days/week and received Allium cepa (3 cc onion juice) and cinnamon (75 mg/kg body weight) daily for 6 weeks by Gavage method.

On the 42nd day Blood samples were collected from the tail vein. At the end of the work, animals were sacrificed by cervical dislocation of the neck.

\section{ELF-EMF Exposure Setup}

The ELF-EMF exposure device used in this study was consisted of two main parts, the radiation area and a transformer. The radiation area was a cylindrical chamber that an electric and a magnetic field were produced there based on the Helmholtz coils. A pair of cooper coils was placed in radiation area one above the other. Coils were separated by the distant of $50 \mathrm{~cm}$ which adjusted to produce a time varying current of $50 \mathrm{~Hz}$ results the average ELF electric field of $1.01 \times 10^{3} \mathrm{~V} / \mathrm{m}$ and the magnetic field flux density of $3 \mathrm{mT}$. Magnetic field strength was $0.24 \mathrm{~A} / \mathrm{m}$.

Cages were placed between the coils where a highly homogeneous electric and magnetic field were produced.

The transformer was used to control input and output voltage and current. The stability of voltage and frequency in ELF-EMF exposure system used in this study is similar to voltage and frequency characteristics of power line Distributions. The output voltage was $25 \mathrm{VAC}$ with stability of $10 \% \max$.

Electric and magnetic field measured in certain radiuses at 8 locations and the average was reported. To avoid temperature changes, a fan was placed in chamber (radiation area). Fluorescent light distant from the device was $3 \mathrm{~m}$ (Table 1, Figure 1).

FSH, LH and Estrogen Hormones Levels Measurement Serum levels of follicle stimulating hormone (FSH), luteinizing hormone $(\mathrm{LH})$ and progesterone were assayed by two-site chemiluminescence (sandwich) immunoassay (16).

Serum Total Antioxidant Capacity Measurement Serum TAC measurement is performed by means of a commercial kit (Randox Co., England). In this measurement, incubation of 2,2'-Azinobis-(3-ethylbenzothiazoline-6-sulphonate) (ABTS) with a peroxidase (methmyoglobin) and hydrogen peroxide was done to produce the radical cation ABTS+. ABTS+ is colorimetrically bluegreen, radical cation which is measured at $600 \mathrm{~nm}$ (17).

Data analysis

Statistical analysis was done using the Analysis of variance

Table 1: ELF-EMF Exposing Parameters ${ }^{a}$

\begin{tabular}{|c|c|c|c|c|}
\hline Duration of exposure & Frequency (Hz) & $\begin{array}{l}\text { Electric field strength, } \\
(\mathrm{V} / \mathrm{m})\end{array}$ & $\begin{array}{l}\text { Magnetic field } \\
\text { strength, } B(A / m)\end{array}$ & $\begin{array}{l}\text { Magnetic field strength, } \\
\mathrm{H}(\mathrm{mT})^{\mathrm{b}}\end{array}$ \\
\hline $\begin{array}{l}4 \mathrm{~h} / \text { day } \\
\text { For } 6 \text { weeks }\end{array}$ & 50 & $1.01 \times 10^{3} \pm 0.05$ & $24 \times 10^{2}$ & $3 \pm 0.01$ \\
\hline
\end{tabular}


(A)

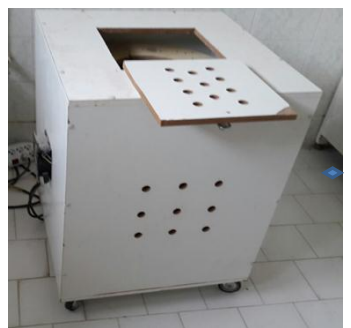

(B)

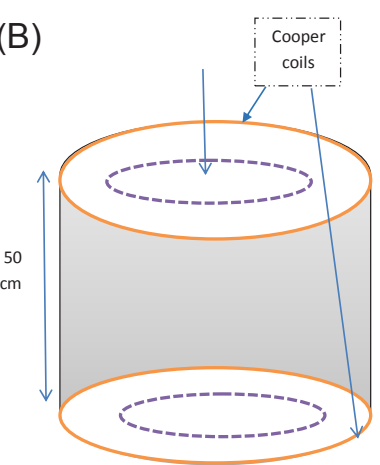

Figure 1. (A) ELF-EMF Producing Device. (B) Diagrammatic Scheme of Radiation Area.

(ANOVA) test for comparison of data in the control group with the experimental groups. The results were expressed as mean \pm SEM (standard error of means). $P$ value less than 0.05 were considered significant.

\section{Results and Discussion}

Electric devices which work by electrical power currents are used both in AC and DC mods. According to the guide issued by the ICNIRP, the direct interaction of external time-varying low frequency electric and magnetic fields could cause alternating electric fields and electrical currents inside the body.

At 50-60 Hz, common time-varying (AC) external electric field of $10^{3} \mathrm{~V} / \mathrm{m}$ will generate an average time-varying electric field of $4 \mathrm{mV} / \mathrm{m}$ within the body. ELF magnetic field strength $(\mathrm{H})$ inside the body is equal to that of outside body due to equality of magnetic permeability $\mu$ of most biological materials with that of free space $\left(4 \pi 10^{7}\right.$ $\mathrm{H} / \mathrm{m})$.

Many studies have been indicated the relationship between ELF EMF and free radicals and ROS production in body (18). ELF EMF exposure may be harmful by impairing the antioxidant defenses (19). Results indicated that extremely low magnetic field of $60 \mathrm{~Hz}$ could adversely affect body antioxidant defensive system by ROS production, other than superoxide radicals (20).

\section{Results of TAC Levels Measurement}

TAC level in control and EMF groups was $0.77 \pm 0.05$ and $0.44 \pm 0.05 \mathrm{mmol} / \mathrm{mL}$, respectively. Also, in cinnamon \& Allium cepa and cinnamon \& Allium cepa+ EMF groups, it was $2 \pm 0.020 .61 \pm 0.05 \mathrm{mmol} / \mathrm{mL}$, respectively. One-way ANOVA shows significant differences between experimental groups in comparison to control group $(P<0.05$;
Table 2).

Results of FSH Levels Measurement

FSH level in control and EMF groups was $1 \pm 0.22$ and $0.6 \pm 0.05 \mathrm{nmol} / \mathrm{mL}$, respectively. Also, in cinnamon \& Allium cepa and cinnamon \& Allium cepa + EMF groups, it was $2.5 \pm 0.05$ and in $1 \pm 0.05 \mathrm{nmol} / \mathrm{mL}$, respectively. ANOVA shows significant differences between experimental groups in comparison to control group $(P<0.05$; Table 2).

Results of LH levels measurement

LH level in control and EMF groups was $0.6 \pm 0.05$ and $0.49 \pm 0.05 \mathrm{nmol} / \mathrm{mL}$, respectively. Also, in cinnamon \& Allium cepa and cinnamon \& Allium cepa + EMF groups, it was $5.5 \pm 0.05$ and $3.3 \pm 0.05 \mathrm{nmol} / \mathrm{mL}$, respectively. One-way ANOVA shows significant differences between experimental groups in comparison to control group $(P<0.05$; Table 2$)$.

\section{Results of Estrogen Levels Measurement}

Estrogen level in control group was $65 \pm 0.01 \mathrm{ng} / \mathrm{land}$ and in EMF group was $51 \pm 0.05$. In cinnamon \& Allium cepa received group was $83 \pm 0.05$ and in cinnamon \& Allium cepa + EMF group was $60 \pm 0.05 \mathrm{ng} / \mathrm{l}$. One-way ANOVA shows significant differences between experimental groups in comparison to control group $(P<0.05$; Table 2$)$. Levels of LH, FSH and estrogen, significantly decreased in second groups that exposed to power frequency EMF of $3 \mathrm{mT}$ and $10^{3} \mathrm{~V} / \mathrm{m}(P<0.05)$ and modified in exposed rats which received Allium cepa and cinnamon $(P<0.05)$. Also, TAC levels were significantly decreased in exposed rats and were moderate in exposed rats received Allium cepa and cinnamon $(P<0.05$; Table 2$)$.

Estrogen regulates gonadotropins release. Also, growth of ovarian follicles ovulation and the number of pre-ovulatory follicles are regulated by secretion of $\mathrm{LH}$ and FSH. Also, production of estrogen and testosterone depend on high LH secretion. It has been indicated that estrogen has significant antioxidant effects at high concentrations (21-24). Levels of LH and FSH were reduced significantly in rats after six weeks of exposure to ELF-EMF of $50 \mathrm{~Hz}$ and 25 $\mu \mathrm{T}$. Results show that effects of ELF-EMF exposure on sex hormones are reversible (25). Also other study showed that exposure to $50 \mathrm{~Hz}$ ELF magnetic (strength of $5 \mathrm{mT}$ ) could affect FSH and LH serum levels (26).

Power frequency EMF exposure may be harmful by impairing the antioxidant defenses. Accurate assessment of

Table 2. Serum Analysis ${ }^{a}$

\begin{tabular}{lllll}
\hline Samples Group & Control & ELF-EMF & Cinamon \& Allium cepa & ELF-EMF Cinamon \& Allium cepa \\
\hline TAC mmol/mL & $0.77 \pm 0.05$ & $0.44 \pm 0.05^{*}$ & $2 \pm 0.02^{*}$ & $0.61 \pm 0.05$ \\
LH nmol/mL & $0.6 \pm 0.05$ & $0.49 \pm 0.05$ & $5.5 \pm 0.05^{*}$ & $3.3 \pm 0.05^{*}$ \\
FSH nmol/mL & $1 \pm 0.22$ & $0.6 \pm 0.05^{*}$ & $2.5 \pm 0.05^{*}$ & $1 \pm 0.05$ \\
Estrogen ng/l & $65 \pm 0.01$ & $51 \pm 0.05^{*}$ & $83 \pm 0.05^{*}$ & $60 \pm 0.05$ \\
\hline
\end{tabular}

Abbreviations: TAC, total antioxidative capacity; $\mathrm{LH}$, luteinizing hormone; FSH, follicle-stimulating hormone.

*Significantly different at $P<0.05$ level (compared with the control group).

${ }^{a}$ Data are presented as mean \pm Standard error (SE). 
induced oxidative stress in biological systems play an important role in investigations which conducted to study role of free radical damages in human pathogenesis TAC data obtained from plasma level is conjured as biomarkers of oxidative stress (27).

ROS production could be affect by ELF-EMF exposure. Based on conducted studies oxidative stress could induce female pathogenesis leading to female fertility and subfertility. ROS could affect physiological functions of female reproduction system tracts and has been implicated in ovarian follicles apoptosis under influence of external chemical and physical factors $(20,28,29)$.

Based on results, Allium cepa and cinnamon could moderate adverse effects of exposure to electromagnetic field on LH and FSH and estrogen level. Hormones levels were significantly increased in exposed rats which received $\mathrm{Al}$ lium cepa and cinnamon.

\section{Conclusion}

Power frequency electromagnetic field could adversely affect sex hormones and total antioxidant capacity levels in exposed rats and Allium cepa and cinnamon could manuscript as an effective pharmacological supplement to moderate exposure degenerative effects.

\section{Ethical Issues}

The ethical committee of Tabriz University of Medical Sciences approved the study.

\section{Conflict of Interests}

All the authors declare that there is no conflict of interest.

\section{Financial Support}

This paper was based on MSc thesis of Elham mansouri with which was supported by the research grant of Women's Reproductive Health Research Center, Tabriz University of Medical Sciences, Tabriz, Iran, with research code 93/2-4/9 and research ethical code 9315 that was approved in 2014.

\section{Acknowledgments}

With special thanks to Women's Reproductive Research Center, Tabriz University of Medical Sciences, Tabriz, Iran for their comments and financial support.

\section{References}

1. Kumar S. Occupational exposure associated with reproductive dysfunctionJ. Occup Health. 2004;46:119. doi:10.1539/joh.46.1

2. Mahram M, Ghazavi M. The effect of extremely low frequency electromagnetic fields on pregnancy and fetal growth and development. Arch Iran Med. 2013;16(4):221-4.

3. Lee S, Park S, Gimm YM, Kim YW. Extremely low frequency magnetic fields induce spermatogenic germ cell apoptosis: possible mechanism. BioMed Res Int. 2014;2014:567183. doi:10.1155/2014/567183.

4. ICNIRP guidelines for limiting exposure to time- varying electric, magnetic and electromagnetic fields up To 300 GHz. Health Physics. 1988;74 (4):494-522.

5. Polk C. Introduction. In: Polk C, Postow E, eds. CRC Handbook of Biological Efects of Electromagnetic Fields. Boca Raton, Florida: CRC Press; 1986:1-24.

6. World Health Organization (WHO). Electromagnetic Fields and Public HealthL - Extremely Low Frequency (ELF). Geneva: WHO; 1998.

7. Röösli M, Jenni D, Kheifets L, Meze G. Extremely low frequency magnetic field measurements in buildings with transformer stations in Switzerland. Sci Total Environ. 2011;409(18):3364-3369. doi:10.1016/j. scitotenv.2011.05.041.

8. Cecconi S, Gualtieri G, Di Bartolomeo A, Troiani G, Cifone MG, Canipari R. Evaluation of the effects of extremely low frequency electromagnetic fields on mammalian follicle development. Hum Reprod. 2000;15(11):2319-2325. doi:10.1093/ humrep/15.11.2319.

9. Agarwal A, Allamaneni SS. Role of free radicals in female reproductive diseases and assisted reproduction. Reprod Biomed Online. 2004;9(3):338. doi:10.1016/s1472-6483(10)62151-7.

10. Wilson BW, Anderson LE, Hilton DI, Phillips RD. Chronic exposure to $60-\mathrm{Hz}$ electric fields: effects on pineal function in the rat. Bioelectromagnetics.1981; 2:371-80. doi:10.1002/bem.2250020408.

11. Asghari A, Montaseri A, Khaki AA. An ultrastructural study of the antioxidant effects of vitamin $\mathrm{E}$ and fennel extract on zona pellucida cell changes of rat ovaries under non-ionizing $50 \mathrm{hz}$ electromagnetic fields. Crescent Journal of Medical and Biological Sciences. 2015;2(2):37-41.

12. Moller P, Wallin H, Knudsen LE. Oxidative stress associated with exercise, psychological stress and life-style factors. Chem Biol Interact. 1996;102:17-36. doi: 10.1016/0009-2797(96)03729-5.

13. Garedaghi Y, Bahavarnia SR. Repairing effect of Allium cepa on Testis degeneration caused by toxoplasma gondii in the rat. Int J Womens Health Reprod Sci. 2014;2(2):080-089.

14. 14. Dhan P, Brahma N. Singh, Garima U. Antioxidant and free radical scavenging activities of phenols from onion (Allium cepa). Food Chemistry, 2007;102 (4):1389-1393.

15. 15. Fathiazad F, khaki A, Nouri M, Khaki A A. Effect of Cinnamon Zeylanicum on serum Testosterone and anti-oxidants levels in Rats. Int J Women's Health Reproduction.2013;1(1):29-35. doi: 10.15296/ ijwhr.2014.12.

16. Hajhosseini L, Khaki A, Merat E, Ainehchi N. Effect of rosmarinic acid on sertoli cells apoptosis and serum antioxidant levels in rats after exposure to electromagnetic fields. Afr J Tradit Complement Altern Med. 2013;10(6):477-480.

17. Khaki A, Fathiazad F, Nouri M, Khaki AA, Khamenehi HJ, Hamadeh M. Evaluation of androgenic activity of allium cepa on spermatogenesis in the rat. Folia 
Morphol (Warsz). 2009;68(1):45-51.

18. Falone S, Mirabilio A, Carbone MC, et al. Chronic exposure to $50 \mathrm{~Hz}$ magnetic fields causes a significant weakening of antioxidant defence systems in aged rat brain. Int J Biochem Cell Biol. 2008;40(12):2762-70. doi: 10.1016/j.biocel.2008.05.022.

19. Li L, Xiong DF, Liu JW, Li ZX, Zeng GC, Li HL. A cross-sectional study on oxidative in workers exposed to extremey low frequency electromagnetic fields. Int J Radiat Biol. 2015;91(5):420-425.

20. Lee BC, Johng HM, Lim JK, et al. Effects of extremely low frequency magnetic field on the antioxidant defense system in mouse brain: a chemiluminescence study. J Photochem Photobiol B. 2004;73(1-2):43-48.

21. Ogunro PS, Bolarinde AA, Owa OO, Salawu AA, Oshodi AA. Antioxidant status and reproductive hormones in women during reproductive, perimenopausal and postmenopausal phase of life. Afr J Med Med Sci. 2014;43(1):49-57.

22. Agacayak E, Basaranoglu S, Tunc SY, et al. Oxidant/ antioxidant status, paraoxonase activity, and lipid profile in plasma of ovariectomized rats under the influence of estrogen, estrogen combined with progesterone, and genistein. Drug Des Devel Ther. 2015;10:9:2975-2982.

23. Tena G, Moran C, Romero R, Moran S. Ovarian morphology and endocrine function in polycystic ovary syndrome. Arch Gynecol Obstet. 2011;284(6):1443-1448.

24. Mumford SL, Browne RW, Schliep KC, et al. Serum antioxidants are associated with serum reproductive hormones and ovulation among healthy women. Nutr. 2016;146(1):98-106.

25. Al-Akhras MA, Darmani H, Elbetieha A. Influence of $50 \mathrm{~Hz}$ magnetic field on sex hormones and other fertility parameters of adult male rats. Bioelectromagnetics. 2006;27(2):127-31.

26. Mostafa RM, Moustafa YM, Ali FM, Shafik A. Sex hormone status in male rats after exposure to 50 $\mathrm{Hz}, 5$ mTesla magnetic field. Archives of Andrology: Journal of Reproductive Systems. 2006;52(5):363369. doi:10.1080/01485010600667092.

27. Young I. Measurement of total antioxidant capacity. J Clin Pathol. 2001;54(5):339. doi:10.1136/jcp.54.5.339.

28. Agarwal A, Aponte-Mellado A, Premkumar BJ, Shaman A, Gupta S. The effects of oxidative stress on female reproduction: a review. Reprod Biol Endocrinol. 2012;10:49. doi:10.1186/1477-7827-1049. doi:10.1186/1477-7827-10-49.

29. Agarwal A, Gupta S, Sekhon L, Shah R. Redox considerations in female reproductive function and assisted reproduction: from molecular mechanisms to health implications. Antioxid Redox Signal. 2008;10(8):1375-403. doi:10.1089/ars.2007.1964.

Copyright $(2016$ The Author(s); This is an open-access article distributed under the terms of the Creative Commons Attribution License (http://creativecommons.org/licenses/by/4.0), which permits unrestricted use, distribution, and reproduction in any medium, provided the original work is properly cited. 